\title{
Rocket observation of energetic electrons in the low-altitude auroral ionosphere during the DELTA campaign
}

\author{
K. Ogasawara*, K. Asamura, T. Takashima, Y. Saito, and T. Mukai \\ The Institute of Space and Astronautical Science, Japan Aerospace Exploration Agency, 3-1-1 Yoshinodai, Sagamihara 229-8510, Japan
}

(Received November 16, 2005; Revised May 21, 2006; Accepted June 6, 2006; Online published September 29, 2006)

\begin{abstract}
This paper reports on properties of energetic electrons observed by the Auroral Particle Detector (APD) on board the sounding rocket S-310-35, which was launched from Andøya Rocket Range, Norway, at 0033:00 UT on 13 December 2004 during the DELTA campaign. The APD was designed to measure energy spectra of energetic electrons in the range of 3.5 to $65 \mathrm{keV}$ every $10 \mathrm{~ms}$ using avalanche photodiodes. The measurement was done at altitudes of 90-140 km (apogee height of the rocket flight), which corresponded to the collisional interaction region of precipitating electrons with the atmospheric constituents. The overall profile of energetic electron precipitations was consistent with auroral images taken from the ground. The downward fluxes almost always exceeded those of upward electrons, and the ratio of downward to upward fluxes increased with energy and also with altitude. This is reasonably understood in terms of the effect of collisions between the energetic electrons and the atmospheric constituents. An interesting feature in energy spectra of precipitating electrons is the existence of non-thermal electrons at higher energies, regardless of inside or outside of auroral arcs. In order to predict the incident downward spectra at the top of the atmosphere, we have applied an analytic method of Luhmann (1976) to evaluate the collisional effect on the electron spectra. As a result, most of the observed energy spectra of precipitating electrons are well expressed by kappa distributions with the thermal energy of a few hundreds of eV and kappa of 5-8, while the spectrum inside a strong arc is better fitted by the sum of a Maxwellian distribution on the lower energy side and a power law at higher energies. To the authors' knowledge, this is the first direct and reliable measurement of energy spectra of electrons in the 10-keV energy range in the auroral ionosphere.
\end{abstract}

Key words: Auroral electron, kappa distribution, medium-energy, non-thermal, sounding rocket, APD.

\section{Introduction}

It is generally accepted that the energy spectra of electrons causing discrete arcs can be expressed by accelerated Maxwellian distributions, while those for diffuse aurora are simple Maxwellian distributions. Actually, however, a deviation from the Maxwellian distribution (either accelerated or non-accelerated) more or less exists at higher energies (for example, see figure 2 of Morooka et al., 2002), though not fully studied. Characteristic features of the energy spectra of auroral electrons are considered to provide information on the source distribution functions in the plasma sheet.

On the other hand, Christon et al. (1988) reported that energy spectra of electrons in the plasma sheet can be well described by kappa distribution functions, of which the lower-energy and higher-energy parts are represented by Maxwellian (thermal) and power-law (non-thermal) distributions, respectively. Hoshino et al. (2001) showed the existence of suprathermal electrons, though the energy range was limited to below $40 \mathrm{keV}$, in association with magnetic

\footnotetext{
*Now at Research Center for Measurement in Advanced Science, Rikkyo University, 3-34-1 Nishi-Ikebukuro, Toshima-ku, Tokyo 171-8501, Japan.

Copyright (c) The Society of Geomagnetism and Earth, Planetary and Space Sciences (SGEPSS); The Seismological Society of Japan; The Volcanological Society of Japan; The Geodetic Society of Japan; The Japanese Society for Planetary Sci-
} ences; TERRAPUB reconnection. Mozer et al. (2002) also showed the enhancement of electrons of a few hundred $\mathrm{keV}$ near the magnetic diffusion region. Although only a limited number of studies have been made, electron measurements covering the thermal to non-thermal energy range are considered to provide an important clue in investigations on the heating and acceleration processes of electrons in the plasma sheet. The thermal to non-thermal transition energy exists at several $\mathrm{keV}$ to several tens of $\mathrm{keV}$.

The purpose of the present paper is to examine the energy spectra of electrons precipitating into the auroral ionosphere in this transition energy range, based on the rocket observation which was carried out during the DELTA campaign (Kurihara et al., 2006). The main objective of the DELTA campaign is to study dynamics of the low-altitude thermosphere during the energy input due to auroral particle precipitations. During this campaign, a sounding rocket, S-310-35, was launched from Andøya Rocket Range, Norway $(69.17 \mathrm{~N}, 16.01 \mathrm{E}$ in geodetic; $67.24 \mathrm{~N}, 113.29 \mathrm{E}$ in geomagnetic), at 0033:00 UT on December 13, 2004. The Auroral Particle Detector (APD) was installed on board the S-310-35 in order to measure energy spectra of precipitating electrons in the energy range of 3.5 to $65 \mathrm{keV}$ every $10 \mathrm{~ms}$ and to provide information on the energy input to the upper atmosphere. The other onboard instruments measured vibrational and rotational temperatures of nitrogen molecules, 
auroral $557.7 \mathrm{~nm}$ intensity, and electron density and temperature. In parallel with the sounding rocket experiment, ground-based observations were made: Fabry-Pérot interferometer in Skibotn and Kiruna for measuring the neutral wind, and EISCAT radar in Tromsø, Kiruna, and Sodankylä for measuring the ion drift and temperature as well as the electron temperature and density (representative examples).

The apogee height of the rocket was $140 \mathrm{~km}$, therefore the altitude range where the measurement was performed corresponded to the collisional interaction region of precipitating electrons with the atmospheric constituents. This effect needs to be taken into account in order to predict the incident precipitating flux at the top of the atmosphere. Different methods and algorithms to solve the electron transport equation in the upper atmosphere have been reported: such as Monte Carlo simulations (Berger et al., 1970; Solomon, 1993, 2001), two-stream calculations (Banks and Nagy, 1970; Nagy and Banks, 1970; Banks et al., 1974; Stamnes, 1980, 1981), and angular dependent or multi-stream calculations (Strickland et al., 1976; Stamnes, 1980; Porter et al., 1987; Lummerzheim et al., 1989). Results of these electron transport calculations have been applied to estimate auroral optical emissions and/or energy spectra of upward electrons above the atmosphere (Lummerzheim et al., 1989). Luhmann et al. (1976) also described a crude method for predicting variations of the energy spectrum of $>1 \mathrm{keV}$ auroral electrons during precipitations into a plane parallel atmosphere with a uniform magnetic field. We apply this method for the prediction of incident energy spectra of electrons, since the electron transport through the atmosphere can be solved analytically with reasonable assumptions.

The following section describes the instrumentation of the APD on board the S-310-35 rocket. Section 3 describes the auroral condition and the observational result of the APD during the rocket flight, while in Section 4, the energy spectra at the top of the atmosphere are discussed, with Section 5 summarizing this paper.

\section{Instrument Description}

The Auroral Particle Detector (APD) on board the sounding rocket S-310-35 was designed to measure energy spectra of electrons ranging from $3.5 \mathrm{keV}$ to $65 \mathrm{keV}$ every $10.24 \mathrm{~ms}$. A key technology in this instrument is to adopt avalanche photodiodes as electron detectors. The avalanche photodiode is a kind of p-n junction semiconductor that has an internal gain due to the avalanche amplification of electrons and holes in the strong electric field within its depletion region. Compared with conventional solid-state detectors (SSDs) which are widely used for measurements of high-energy particles, it can drastically improve the energy resolution in a 10-keV energy range and decrease the lower energy limit of detectable electrons down to several $\mathrm{keV}$ (Ogasawara et al., 2005, 2006).

Four avalanche photodiodes were installed in this instrument, as shown in Fig. 1. The output charge pulse from each avalanche photodiode is converted to the voltage pulse by a preamplifier, followed by a shaping amplifier and A/D conversion for pulse height analysis. The data accumulated for $10.24 \mathrm{~ms}$ were transmitted to the ground through the

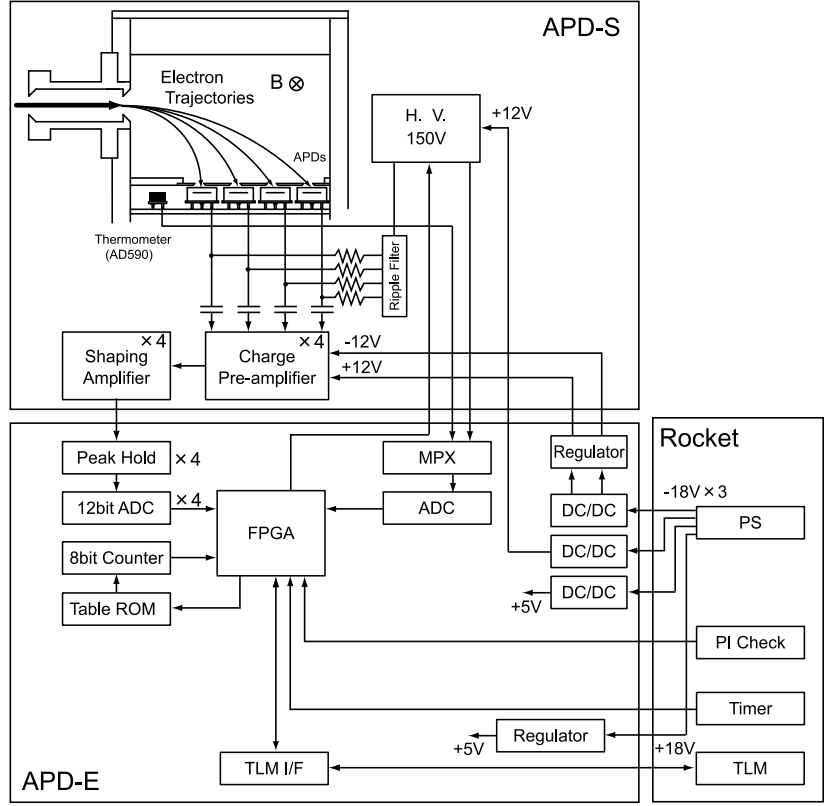

Fig. 1. Schematic diagram of the Auroral Particle Detector onboard the S-310-35 rocket. The system consists of the two parts: the sensor part (APD-S) and the electronics subsystem (APD-E). Sample trajectories of incoming electrons are also shown in the APD-S.
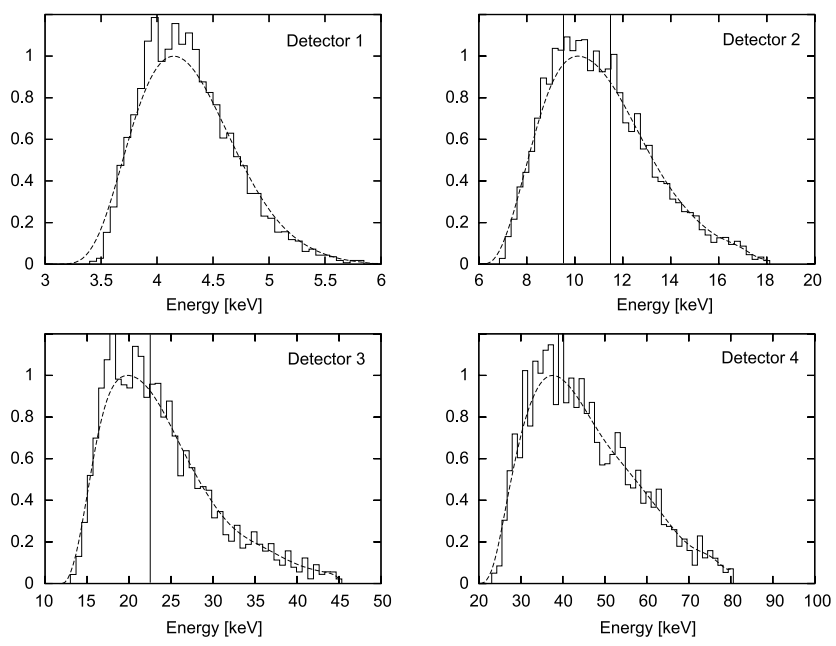

Fig. 2. Calculated transmission curves for four detectors. Calculated values (solid lines) were smoothed with the dashed curves. Vertical lines in the panel of Detector 2 and Detector 3 indicate separations of energy channels.

PCM telemetry. In order to reject incoming photons, trajectories of incident electrons were deflected using a homogenous magnetic field supplied by a permanent magnet. Since electron trajectories are different depending on their energies, four avalanche photodiodes respectively cover their own energy ranges.

Figure 2 shows calculated transmission efficiencies for four detectors, in which the peak for each detector is normalized by a smoothed curve. There is an energy gap (5.2$6.9 \mathrm{keV}$ ) between Detector 1 and Detector 2 due to characteristics of the magnetic field and the detector layout. The avalanche photodiode itself also has the capability to resolve the energy of incident electrons. Consequently, the 

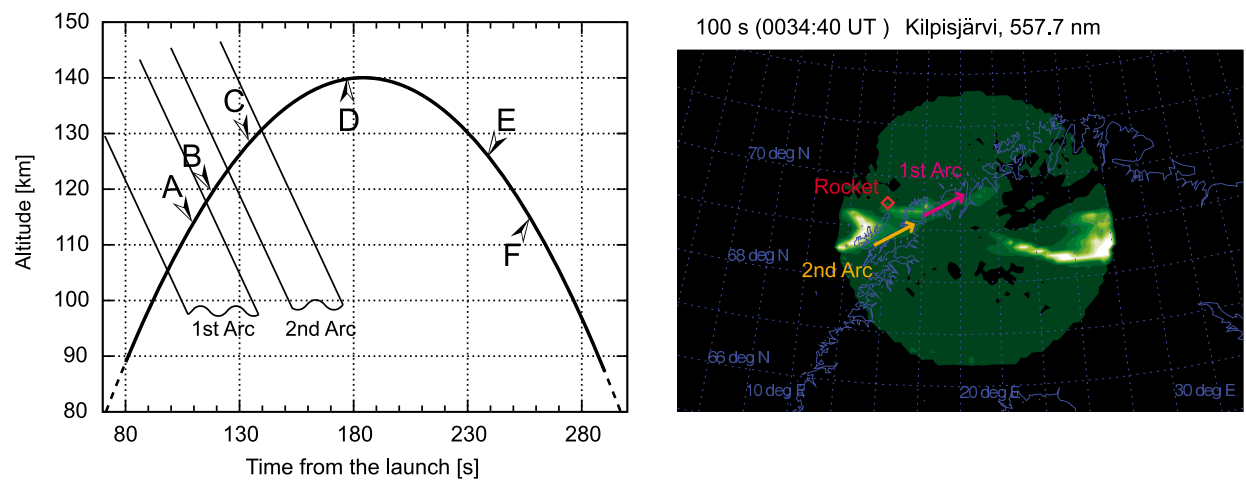

Fig. 3. In the left panel, the altitude profile of the S-310-35 rocket is shown. The APD measurement was performed during a thick solid curve. Marks of A-F show points of the energy spectra discussed in this paper. The right panel shows an auroral image in $557.7 \mathrm{~nm}$ taken from Kilpisjärvi at 100 $s$ after the launch. The position of the rocket is shown by a red square.

Table 1. Energy channels of the APD onboard S-310-35. Center energies are calculated taking into account the response curves as shown in Fig. 2. Note that there are overlapping energies between neighbor channels due to the limitted energy resolution of the APD.

\begin{tabular}{ccc}
\hline $\begin{array}{c}\text { Detector } \\
\text { No. }\end{array}$ & $\begin{array}{c}\text { Center energy } \\
\mathrm{keV}\end{array}$ & $\begin{array}{c}\text { Energy range } \\
\mathrm{keV}\end{array}$ \\
\hline 1 & 4.3 & $3.5-5.2$ \\
2 & 8.5 & $6.9-9.7$ \\
2 & 10.4 & $8.9-12.0$ \\
2 & 13.3 & $11.1-15.8$ \\
3 & 18.8 & $14.7-24$ \\
3 & 28.6 & $21-36$ \\
4 & 45.1 & $25-65$ \\
\hline
\end{tabular}

whole energy range is divided into seven energy channels (Table 1).

The total energy geometrical factor of the spectrometer is calculated to be $6.9 \times 10^{-3} \mathrm{~cm}^{2}$ str keV. The field of view covers $22^{\circ}$ for the azimuthal angle and $8^{\circ}$ for the elevation angle (i.e. pitch angle resolution of this instrument), whose center is directed perpendicularly to the rocket spin axis. The angle between the geomagnetic field and the rocket spin axis during the flight was about $20^{\circ}$, and thus this instrument covered pitch angles of $70-110^{\circ}$.

\section{Observation}

\subsection{Overview}

The S-310-35 sounding rocket was launched to the northward from Andøya Rocket Range, Norway (69.17 N, 16.01 $\mathrm{E}$ in geodetic; $67.24 \mathrm{~N}, 113.29 \mathrm{E}$ in geomagnetic), at 0033:00 UT on December 13, 2004, into the auroral precipitation region. The left-hand panel in Fig. 3 shows an altitude profile of the rocket trajectory with time. The apogee of the rocket flight was $140 \mathrm{~km}$ at $184 \mathrm{~s}$ from the launch, while the total flight time was $350 \mathrm{~s}$. The APD electron measurement was carried out at altitudes of 90-140 km, as shown by a thick curve. Throughout the flight, the background noise was negligibly small, as had been intended in the instrument design. Marks of A through F represent times for detailed investigation of the energy spectra. The right-hand panel in Fig. 3 shows an auroral image of $557.7 \mathrm{~nm}$ taken from Kilpisjärvi (69.0 N, $20.9 \mathrm{E})$ at $100 \mathrm{~s}$ (0034:40 UT) from the launch. Two major arcs can be seen around the rocket (marked by the red square), projected at an altitude of $100 \mathrm{~km}$ along the geomagnetic field lines. These two arcs are passed through by the rocket, as shown by arrows in the figure. The second arc looks brighter than the first one. Two arcs crossed by the sounding rockets are schematically shown in the left panel from $100 \mathrm{~s}$ to $140 \mathrm{~s}$. After the two arcs were passed through the rocket trajectory, no discrete arc was found near the rocket. All images taken from Kilpisjärvi during the flight are shown in figure 1 of Kurihara et al. (2006).

Figure 4 shows an energy-time spectrogram of all measured electrons with pitch angles of $70-85^{\circ}$ and $95-85^{\circ}$ during the whole flight. Data of the pitch angle range of $85-95^{\circ}$ are omitted to remove artificial noises possibly due to NTV (Nitrogen Temperature Instrument) (Kurihara et al., 2006). Each spectrum is averaged over one spin period ( $\sim 0.9 \mathrm{~s})$. Intense precipitations can be seen between $98 \mathrm{~s}$ and $139 \mathrm{~s}$. After this time, the electron flux became weaker. In more detail, the region where intense fluxes are seen is largely divided into two parts (between $98 \mathrm{~s}$ and $115 \mathrm{~s}, 125 \mathrm{~s}$ and $139 \mathrm{~s}$ ), though the flux intensity fluctuates with time. The latter enhancement of the electron flux seems more intense. These features are quite consistent with those of the auroral arcs passing through the rocket in the images taken from Kilpisjärvi.

Figure 5 shows profiles of downward and upward electron fluxes at four lower-energy channels. The red and blue solid lines show electron fluxes of the downward $\left(70-85^{\circ}\right)$ and upward $\left(95-110^{\circ}\right)$ pitch angles, respectively. First of all, the difference in the precipitating fluxes between the first and second arcs is clearly seen, and the fluxes corresponding to the second arc are almost tenfold larger than those of the first arc. The maximum flux at the lowest energy channel reached close to $10^{8}\left(\mathrm{~cm}^{2} \mathrm{~s} \text { str keV}\right)^{-1}$ during the strong precipitation. Simultaneously, some fine structures are also seen during this time period, probably corresponding to temporal and/or spatial variations of auroral arcs. Thereafter, in the weaker precipitation, the differential fluxes stayed in the range of $10^{5}-10^{6}\left(\mathrm{~cm}^{2} \mathrm{~s} \mathrm{str} \mathrm{keV}\right)^{-1}$ on the average. It is also clearly seen that the downward fluxes exceeded the upward ones almost throughout the flight. This fact indicates that the observed electron fluxes remain anisotropic in spite of frequent collisions in the dense atmo- 


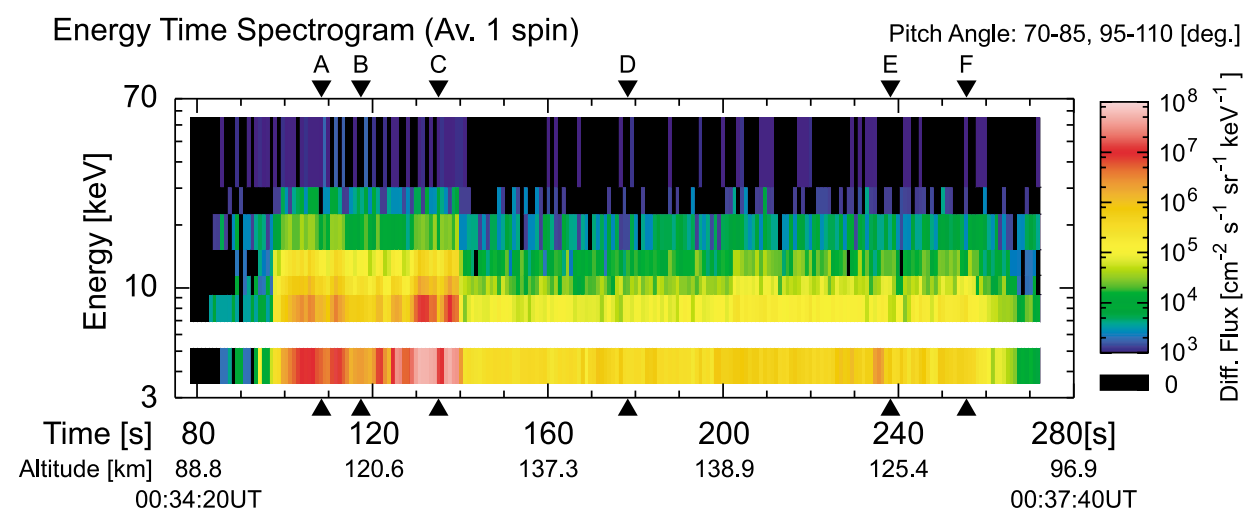

Fig. 4. Energy-time spectrogram of electrons during the flight of S-310-35 averaged over one spin period ( $\sim 0.9 \mathrm{~s})$. The color bar gives differential fluxes of the electrons on a logarismic scale. The gap between 5.2 and $6.9 \mathrm{keV}$ indicates no data because of the geometrical characteristics of the instrument.

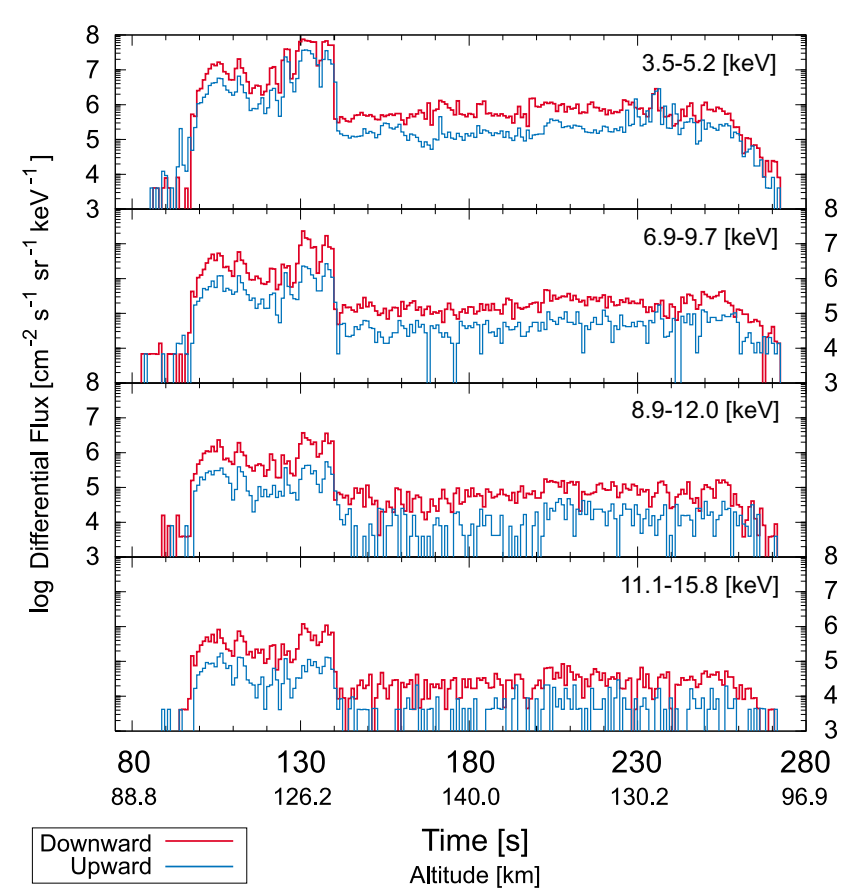

Fig. 5. Temporal profile of differential fluxes at four energy channels averaged over one spin period; from the top, at energies of $3.5-5.2 \mathrm{keV}$ (4.3 keV), 6.9-9.7 keV (8.5 keV), 8.9-12.0 keV (10.5 keV), 11.1-15.8 $\mathrm{keV}(13.5 \mathrm{keV})$, where the energy in parentheses shows the center energy of each channel. Red and blue lines show downward and upward fluxes, respectively.

sphere even at these low altitudes. The more frequent the collisions become, the more isotropic the electron distribution would be under a condition of the homogeneous magnetic field from 90 to $140 \mathrm{~km}$. Figure 6 shows ratios of the downward to upward electron fluxes at the selected lowerenergy channels. The ratio becomes larger with increasing energies, and in the lowest energy range (3.5-5.2 keV), it is highest near the apex (180 s), while the altitude variations at other higher energies are smaller. These characteristics are reasonably understood, considering the energy dependence of the effect of the atmospheric absorption; higher-energy electrons can penetrate into the lower-altitude atmosphere with less energy loss.

Figure 7 shows energy spectra averaged over ten spin pe-

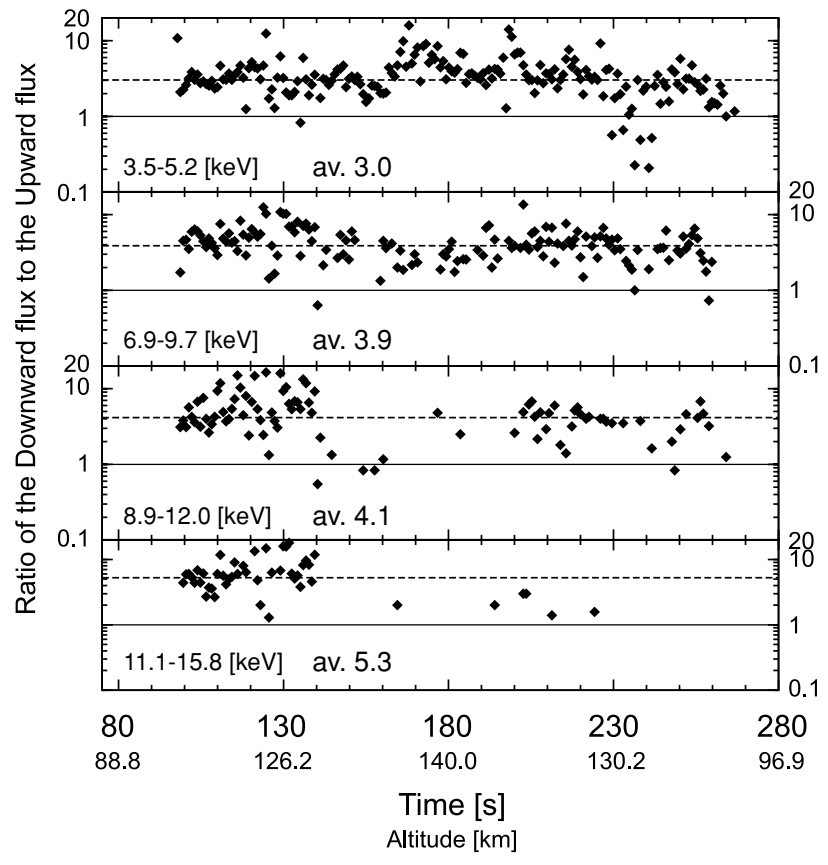

Fig. 6. Ratios of the downward to upward fluxes in four energy ranges (see the caption in Fig. 5). A horizontal dashed line in each panel represents the geometric mean of the ratio.

riods at the observation points from $\mathrm{A}$ to $\mathrm{F}$, where solid and dashed curves show energy spectra of the downward (70$\left.85^{\circ}\right)$ and the upward $\left(95-110^{\circ}\right)$ pitch angles, respectively. Thin dotted lines denote one count levels (the lowest level for detection) during the integrated time. Here, A (104$113 \mathrm{~s}, 113 \mathrm{~km})$ and $\mathrm{C}(130-139 \mathrm{~s}, 129 \mathrm{~km})$ represent the points inside the two auroral arcs: $\mathrm{A}$ indicates the first arc and $\mathrm{C}$ indicates the second one. $\mathrm{B}(113-122 \mathrm{~s}, 119 \mathrm{~km})$ is chosen as a point between the two arcs. D, E, and F represent the weak precipitation region far away from the arcs: D (174-182 s, $140 \mathrm{~km})$ corresponds to the apex of the flight, and $E(234-243 \mathrm{~s}, 126 \mathrm{~km})$ and $\mathrm{F}(252-260 \mathrm{~s}, 116 \mathrm{~km})$ are chosen from the downleg of the rocket. The fluxes around the auroral arcs (A to C) are significantly more intense than those away from the $\operatorname{arcs}(\mathrm{D}$ to $\mathrm{F}$ ). It is evident that the downward fluxes always exceed the upward ones. The effect of the atmospheric absorption is more significant as the 

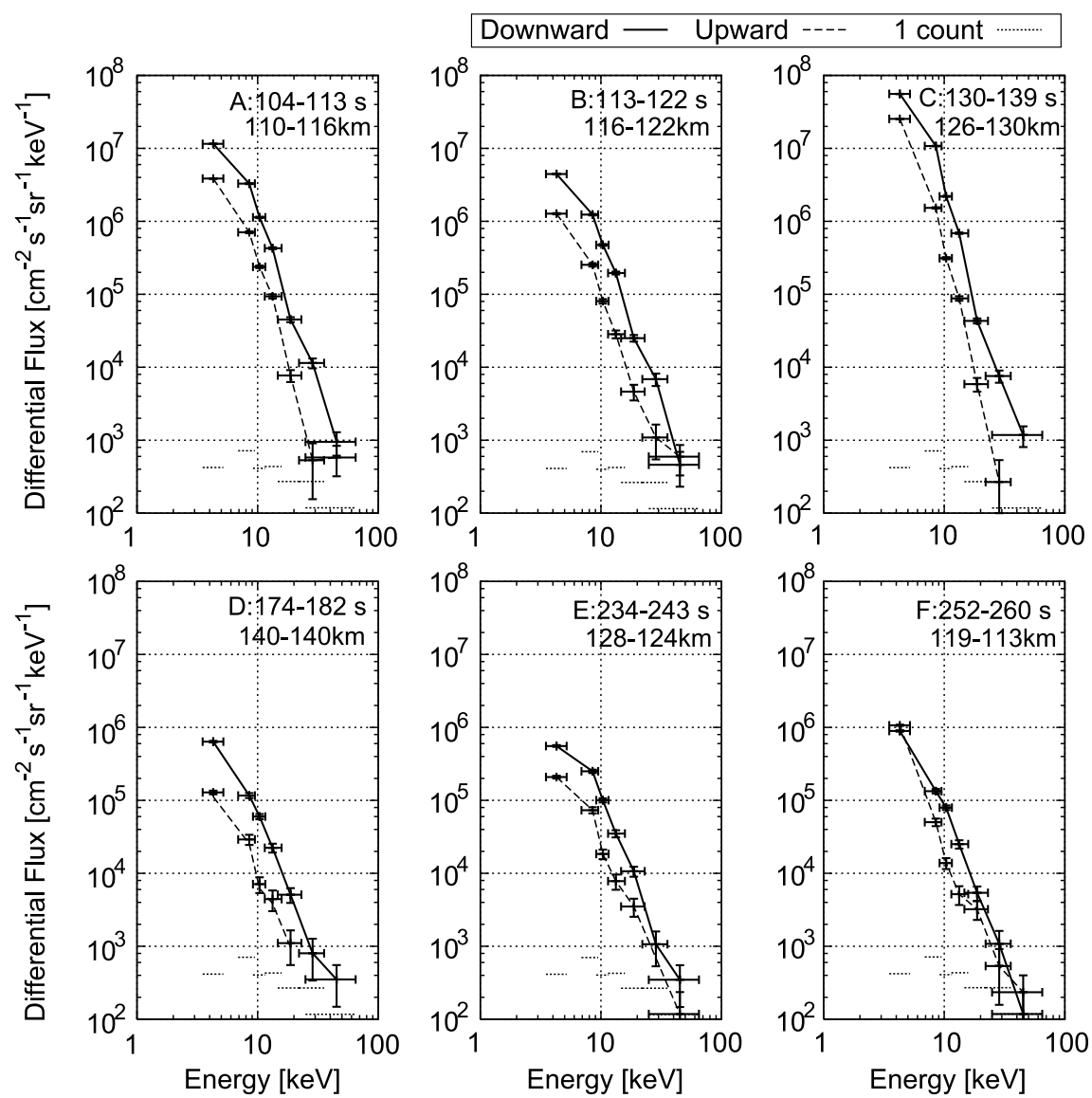

Fig. 7. Observed downward (solid) and upward (dashed) electrons averaged over ten spin periods. Thin dotted lines corresponding to ' 1 count' indicate the one count level during the integrated period.

altitude decreases. It is also seen in all of the cases that the energy spectra show the existence of non-thermal electrons at energies higher than $10 \mathrm{keV}$. As mentioned before, the energy spectra of precipitating electrons have the information on their original spectra in the source region and the effect of the atmospheric absorption. Further discussion on the energy spectra is given in the next section.

\section{Discussion}

\subsection{Energy spectra of the downward electrons}

Because the observation was done in the ionospheric E-region, collisional interactions between the precipitating electrons and the atmospheric constituents were quite frequent. MacDonald and Walt (1961) derived a transport formula from the time independent Fokker-Planck equation that describes the transport of a uniform primary electron beam of infinite extent in a plane scattering medium and a diverging magnetic dipolar field. The Fokker-Planck equation is derived from the Boltzmann equation and their result is expressed in the form:

$$
\begin{aligned}
\mu v \frac{\partial f}{\partial x} & +\frac{3 v}{2\left(R_{e}+r\right)}\left(1-\mu^{2}\right) \frac{\partial f}{\partial \mu} \\
& =\frac{1}{2} \frac{\partial}{\partial \mu}\left[\frac{\left\langle\Delta \mu^{2}\right\rangle}{\Delta t} \frac{\partial f}{\partial \mu}\right]-\frac{1}{\rho} \frac{\partial}{\partial E}\left[\frac{\langle\Delta E\rangle}{\Delta t} f\right] .
\end{aligned}
$$

Two approximations are then made in Eq. (1). Firstly, pitch angle scatterings of the primary electrons (the first term on the right hand) are neglected. Especially in the case of precipitating electrons of $>1 \mathrm{keV}$, collisions in the atmosphere are due mainly to ionizations of the atmospheric atoms or molecules. The ionization causes little scattering in pitch angles of the incident electrons, and hence the pitch angle scattering can be reasonably neglected. Secondly, a geomagnetic mirror force (the second term on the left hand) is neglected, because the magnetic field is almost constant in the altitude range under consideration $(90-300 \mathrm{~km})$. These assumptions can simplify the electron transport equation into the first-order partial differential equation as follows;

$$
\mu v \frac{\partial f}{\partial x}=-\frac{1}{\rho} \frac{\partial}{\partial E}\left[\frac{\langle\Delta E\rangle}{\Delta t} f\right]
$$

where $f=f(\mu, r, E)$ is the number of electrons per unit volume, unit pitch angle, and unit energy, $\mu$ is cosine of the pitch angle, $v$ is the electron velocity corresponding to its energy, $r$ is the vertical position from the surface of the earth, and $\rho$ is the mass density of the atmosphere. The symbol $x$ is called atmospheric depth in the unit of $\mathrm{g} / \mathrm{cm}^{2}$, as defined by

$$
x(r)=\int_{r}^{r_{\infty}} \rho(r) d r .
$$

Here, $r_{\infty}$ represents the top of the atmosphere, where effects of the atmospheric loss are negligible. We have applied the NRL MSIS-E-00 atmospheric model for the mass density in Eq. (3). If the particle range in the atmosphere is given 
by an analytic form in Rees (1963), Eq. (2) is solved in the way of Luhman (1976). If the differential flux at the top of the atmosphere (defined here as $300 \mathrm{~km}$ from the ground) is defined as $J_{0}(E)$ (electrons $/ \mathrm{cm}^{2} \mathrm{~s}$ str $\mathrm{keV}$ ), the electron distribution $f(\mu, x, E)$ at the atmospheric depth $x$ is written in the form (MacDonald and Walt, 1961),

$$
f(\mu, x, E)=\frac{2 \pi}{v}\left(\frac{E}{\xi}\right)^{0.75} J_{0}(\xi),
$$

where $\xi$ is defined in the form,

$$
\xi(\mu, x, E)=\left[\frac{1}{A}\left(\frac{x}{\mu}+A E^{1.75}\right)\right]^{1 / 1.75} .
$$

The constant $A$ is defined in Rees (1963) as $A=4.57 \times$ $10^{-6}$. Figure 8 shows the energy at the altitude of $300 \mathrm{~km}$ versus several altitudes calculated by Eq. (5). It is seen that an altitude range from $100 \mathrm{~km}$ to $140 \mathrm{~km}$ has a significant effect on the energy of precipitating electrons under $10 \mathrm{keV}$ in terms of the energy loss by collisions with atmospheric atoms. The derivation of Eq. (4) is given in the Appendix. The distribution $f$ can be converted to the flux $J$ with adjusting its unit to (electrons $/ \mathrm{cm}^{2} \mathrm{~s}$ str $\mathrm{keV}$ ),

$$
J=\frac{v}{2 \pi t(\mu)} f,
$$

where $t(\mu)$ is the proportion of the solid angle that is occupied by the unit angle around $\mu$ to the whole downward hemisphere $(2 \pi)$. Then, we can fit $J$ to the measured spectrum with parameters of $J_{0}$, which stands for the incident

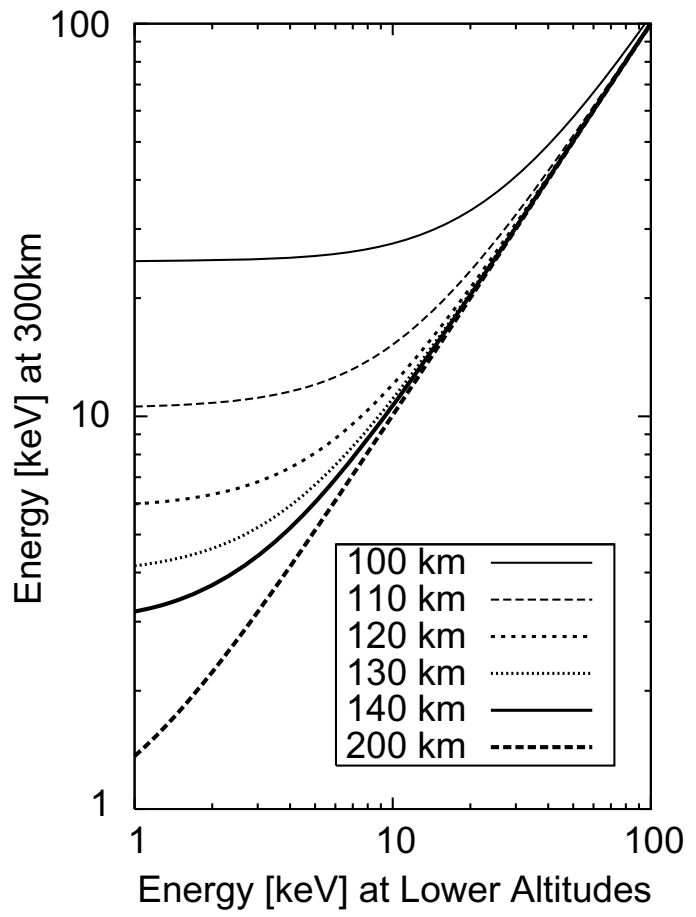

Fig. 8. Relation of energies of precipitating electrons between $300 \mathrm{~km}$ and lower altitudes calculated by Eq. (5) for the pitch angle of $78^{\circ}$. The altitude of $300 \mathrm{~km}$ is assumed to be the top of the atmosphere. The atmospheric depth is calculated from the atmospheric model NRL MSIS-E-00 spectrum of the precipitating electrons at the top of the atmosphere.

We have applied three types of distribution functions in order to fit the observational data: Maxwellian, kappa, and power law distribution. The Maxwellian distribution is expressed by the following form,

$$
J_{0} \propto E \cdot \exp \left(-\frac{E}{E_{T}}\right) .
$$

The kappa distribution exhibits a long tail which strongly deviates from a Maxwellian distribution (thermal) at higher energies. The tail is asymptotically modeled by the power law distribution (non-thermal) with its power of $\kappa$. The kappa distribution is expressed in the form,

$$
J_{0} \propto E \cdot\left(1+\frac{E}{\kappa E_{T}}\right)^{-(\kappa+1)} .
$$

For higher energy electrons (typically over $10 \mathrm{keV}$ ), a power law distribution is sometimes applicable, as defined in the form,

$$
J_{0} \propto E^{-\gamma}
$$

where $\gamma$ denotes the power of the spectrum.

In Fig. 9, the observed downward spectrum for case A (104-113 s) is fitted by both the Maxwellian distribution and the kappa distribution for comparison. The solid

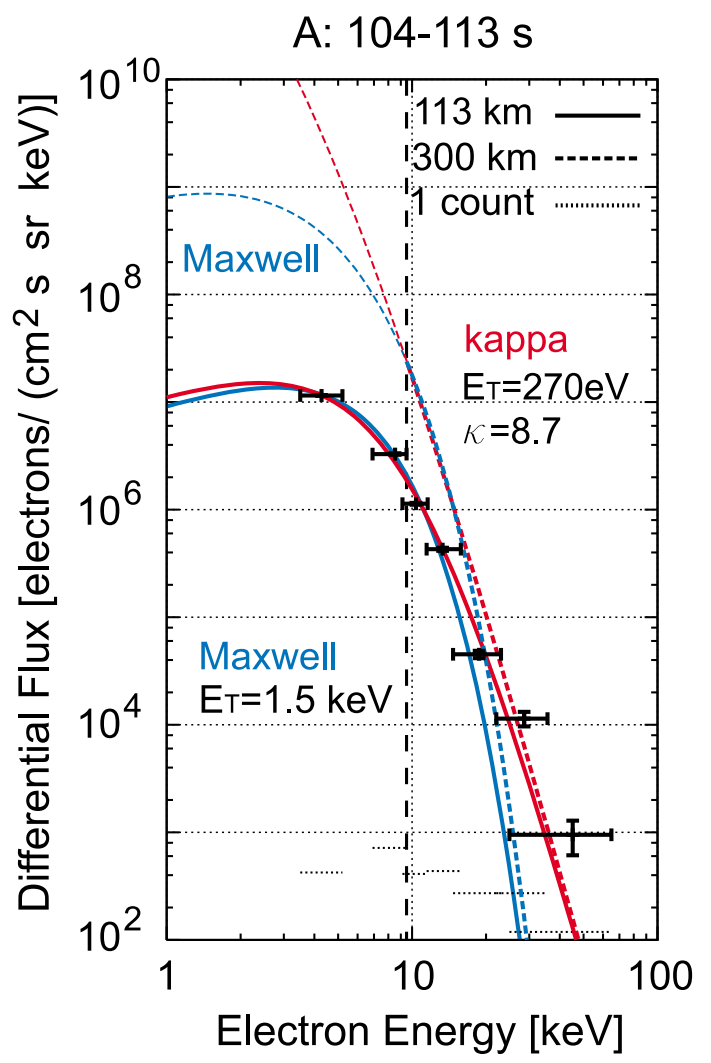

Fig. 9. Result of fitting by a kappa distribution (red) and a Maxwellian distribution (blue). The solid curves show the fitted spectra to the observed one, and the dashed curves show the anticipated flux at the top of the atmosphere. The difference between the solid and dashed curves was due to the atmospheric absorption. The calculated flux at $300 \mathrm{~km}$ may not be meaningful at the energy range to the left of the dashed line (see text). 


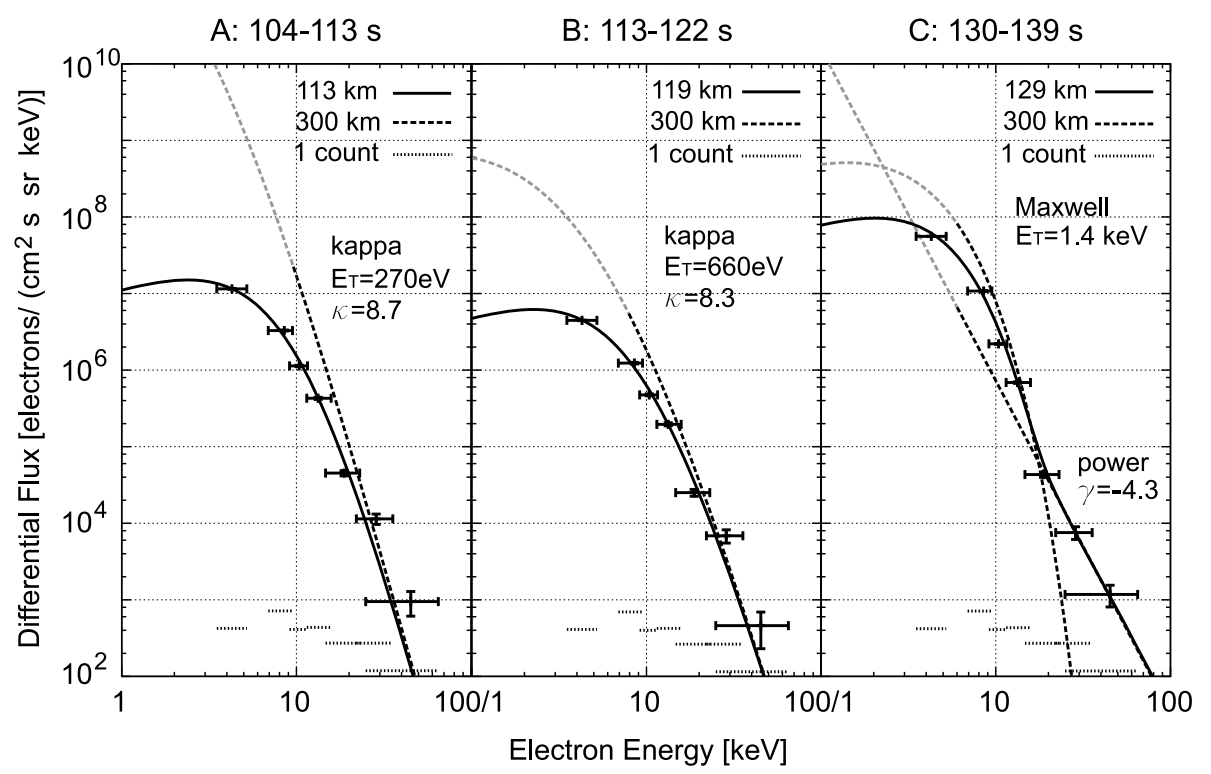

Fig. 10. Fitted energy spectra of downward electrons close to the arcs. The solid curves show the observed data, while the dashed curves are the assumed spectra at the top of the atmosphere $(300 \mathrm{~km})$. The gray part of the dashed curve may not be meaningful. The border of the gray part corresponds to $9.8 \mathrm{keV}$ in $\mathrm{A}, 7.8 \mathrm{keV}$ in $\mathrm{B}$ and $6.2 \mathrm{keV}$ in $\mathrm{C}$ (see Fig. 8).

curves show the fitted spectra to the observed data, while the dashed curves show the assumed spectra at the top of the atmosphere (altitude of $300 \mathrm{~km}$ ). The blue and red curves show the Maxwellian and kappa distributions, respectively. The Maxwellian distribution with a thermal energy of 1.5 $\mathrm{keV}$ is well fitted in the lower energy part $(\leq 15 \mathrm{keV})$, but in the higher energy part, drops down steeper than the observed spectrum. Instead, the 'non-thermal' tail is well fitted by the kappa distribution function, which can also be fitted to the energy distribution in the whole energy range. In this case, the thermal energy of the kappa distribution is $270 \mathrm{eV}$ and the derived $\kappa$ is 8.7. The difference in the flux between $300 \mathrm{~km}$ and the observational altitude becomes less as the energy increases. This is reasonably understood in terms of the atmospheric absorption depending on the electron energy. Precipitating electrons lose their energies through the ionization of the atmospheric constituents during the transport from the top of the atmosphere to the observation point. According to the energy-loss calculation taking account of the pitch angles of $78^{\circ}$, the measured lowest energy of $4.3 \mathrm{keV}$ at the observation point corresponds to $9.8 \mathrm{keV}$ (vertical dashed line) at $300 \mathrm{~km}$ (see Fig. 8). Therefore, the calculation of the fitted spectrum at an altitude of $300 \mathrm{~km}$ may not be meaningful below this energy (dashed line).

\subsection{Around the arcs}

Figure 10 summarizes the fitting results of energy spectra observed close to the $\operatorname{arcs}(\mathrm{A}, \mathrm{B}, \mathrm{C})$. The vertical axes show differential fluxes of the downward electrons, and the horizontal axes show the electron energy. The spectra were averaged over ten spin periods to obtain sufficient counting statistics in higher energy channels. The dashed curves show the anticipated spectra at the top of the atmosphere, while the solid lines are the fitted spectra at the altitude of the observation. The gray part of each fitted spectrum for the top of the atmosphere may not be meaningful because of the atmospheric energy loss, as explained before. As was discussed in the former section, the kappa distribution function $\left(E_{T}=270 \mathrm{eV}\right.$ and $\left.\kappa=8.7\right)$ is drawn in case A. In case $B$, between two arcs, the thermal energy of the kappa distribution is $660 \mathrm{eV}$ and $\kappa$ is 8.3. The flux in B was relatively lower than those inside the auroral arcs (A and C). In case $C$, the kappa distribution could not be well fitted because of an abrupt bending in the observed spectrum around $20 \mathrm{keV}$, and a two-component distribution function is more applicable. For this case, the sum of a Maxwellian distribution (Eq. (7)) and a power law distribution (Eq. (9)) is chosen instead. The thermal energy of the Maxwellian is fitted to $1.4 \mathrm{keV}$. The spectrum above the energy of $20 \mathrm{keV}$ is well fitted by the power law, whose power is 4.3 .

The distribution functions of electrons above the discrete aurora are generally fitted by the accelerated Maxwellian distributions, suggesting the existence of a parallel electric field in a higher-altitude acceleration region. In the present observation, we have fitted non-accelerated distributions (Kappa or Maxwellian) to the observed spectra, but there is a possibility of the parallel acceleration below several $\mathrm{keV}$. However, it is difficult to determine the parallel acceleration potential uniquely, because the measured energy range is higher than usual acceleration energies $(<\mathrm{sev}-$ eral $\mathrm{keV}$ ), and any acceleration energy near and below the measured energy range could be fitted to the observed spectrum. Moreover, the atmospheric loss significantly deforms the distribution function at energies lower than several $\mathrm{keV}$ (see Fig. 8), therefore the peak would become obscure at the rocket altitude.

\subsection{Far from the arcs}

Figure 11 shows the electron spectra observed far away from the arcs, which were also averaged over ten spins. As discussed earlier, the precipitation became relatively weak in this region. Similar to the cases around the arcs, nonthermal tails in the energy spectra are clearly seen, so that 


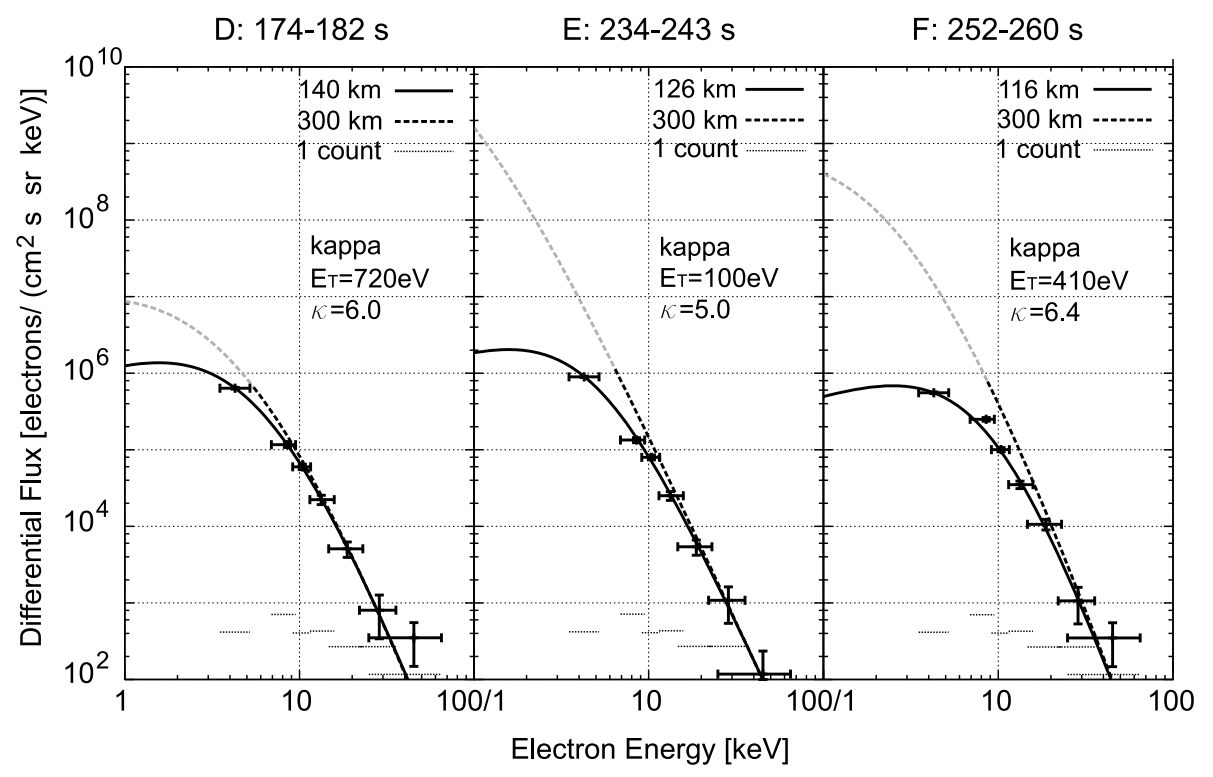

Fig. 11. Fitted energy spectra of downward electrons far away from the arcs, in the same format as Fig. 10. The border of the gray part corresponds to $5.4 \mathrm{keV}$ in $\mathrm{D}, 6.6 \mathrm{keV}$ in $\mathrm{E}$ and $8.6 \mathrm{keV}$ in $\mathrm{F}$ (see Fig. 8).

the kappa distribution functions are valid in the whole energy range. In case $\mathrm{D}$, the thermal energy of the kappa distribution is $720 \mathrm{eV}$ and $\kappa$ is 6.0. This case was observed near the apex, and the effect of the atmospheric absorption would be the smallest. In case $\mathrm{E}$, the thermal energy of the kappa distribution is $100 \mathrm{eV}$ and $\kappa$ is 5.0. In case $\mathrm{F}$, the thermal energy of the kappa distribution is $410 \mathrm{eV}$ and $\kappa$ is 6.4. The value of $\kappa$ is a little smaller in $\mathrm{D}, \mathrm{E}$, and $\mathrm{F}$ than in the cases A and B. Note, however, that the parameters in D, $\mathrm{E}$, and $\mathrm{F}$ are quite similar to those of the plasma sheet electrons reported by Christon et al. (1988), and it is reasonable since the magnetic field lines of the nightside auroral region are linked to the plasma sheet.

\section{Concluding Remarks}

The Auroral Particle Detector was installed on the sounding rocket S-310-35 launched from Andøya in Norway on December 13, 2004, and successfully measured auroral electrons of 3.5-65 keV. A key technology in this instrument is to adopt avalanche photodiodes as the electron detectors. Compared to conventional SSDs, the utilization of avalanche photodiodes (APDs) can drastically improve the energy resolution in a $10-\mathrm{keV}$ energy range and decrease the lower energy limit of detectable electrons down to several keV. Moreover, the reliability of the detection efficiency in this energy range is significantly improved. This experiment is the world's first trial of applying the APDs to the measurement of low-energy electrons in space, and the results have adequately demonstrated the effectiveness of the APDs. The measurement was made at the altitude of 90-140 km, where collisions between the precipitating electrons and the atmospheric constituents occur frequently. The effect of the atmospheric absorption is evident in the ratio of the upward to the downward fluxes. There were strong precipitations from 100-104 s from the launch, which agreed well with the ground-based auroral observations. The energy spectra of the downward electrons are dis- cussed, considering the effect of the atmospheric absorption with the method of Luhmann (1976). Around auroral arcs, non-thermal tail features in the electron spectra were found, and the kappa distribution functions are well fitted to the spectra. The two-component distribution function (the sum of a Maxwellian distribution and a power law) was identified in one case ( $\mathrm{C}$ in Fig. 10). In cases far from the arc, non-thermal tails are also evident and the distribution functions are well fitted by kappa distributions $(\kappa=5 \sim 8)$. The kappa distribution is consistent with the spectra of the plasma sheet electrons reported in Christon et al. (1988).

Acknowledgments. The authors wish to thank Y. Ogawa at STEL and K. Kauriste at FMI for providing auroral images. We are also deeply grateful to H. Matsumoto and T. Goka in the Institute of Aerospace Technology of JAXA for providing the electron beam facility for the calibration of the instrument. We also wish to thank members of the DELTA campaign, especially for T. Abe and J. Kurihara. This work was partly supported by the Yamada Science Foundation, the Grant-in-Aid for Exploratory Research (15654083) and the Grant-in-Aid for JSPS Fellows (1511586).

\section{Appendix. Derivation of Eq. (4)}

Equation (2) is derived from Eq. (1) with two assumptions: the magnetic converging term is neglected, and pitch angle scattering is neglected (i.e. $\mu$ maintains constant). Then, in Eq. (2), the Fokker-Planck coefficient $\langle\Delta E\rangle / \Delta t$ can be expressed in terms of the stopping power $d E / d s$ along the electron trajectory $\mathrm{s}\left(\mathrm{g} / \mathrm{cm}^{2}\right)$,

$$
\frac{\langle\Delta E\rangle}{\Delta t}=v \rho \frac{d E}{d s} .
$$

If $\mathrm{G}$ is defined as

$$
G=v \frac{d E}{d s} f,
$$

Then, Eq. (2) is equivalent to a simple first-order partial differential equation:

$$
\mu \frac{\partial G}{\partial x}+\frac{d E}{d s} \frac{\partial G}{\partial E}=0 .
$$


$\mu$ is constant by the assumption mentioned above, so the characteristic curve of Eq. (A.3) is derived as

$$
\frac{d x}{\mu}=\frac{d E}{(d E / d s)},
$$

which can be integrated using a constant of integration $C$,

$$
\frac{x}{\mu}-\int \frac{d E}{(d E / d s)}=C .
$$

Considering that $G$ is an arbitrary constant from the characteristic curve, a general solution for Eq. (A.3) is then given by

$$
G=\phi(C),
$$

where $\phi$ is an arbitrary function. From the definition by Eq. (A.2), $f$ can be obtained:

$$
f(\mu, x, E)=\phi(C) /\left(v \frac{d E}{d s}\right) .
$$

The energy spectra at the top of the atmosphere $(x=0)$ is given by $J_{0}(E)$ in electrons $/\left(\mathrm{cm}^{2} \mathrm{~s}\right.$ str $\left.\mathrm{keV}\right)$. Assuming an isotropic distribution over the downward hemisphere, $f$ and $J_{0}$ are related by

$$
\int_{0}^{1} f(\mu, 0, E) d \mu=\frac{2 \pi}{v} J_{0}(E),
$$

that gives a boundary condition. The integral in the second term of the left part in Eq. (A.5) resembles the well-known definition of the range integral for charged particles in matter:

$$
-\int_{E_{0}}^{0} \frac{d E}{(d E / d s)}=R\left(E_{0}\right),
$$

where $R\left(E_{0}\right)$ is the range of a particle of initial energy $E_{0}$. Rees (1963) expressed an analytic form of the empirical relationship between the range of electrons in the atmosphere and the initial energy:

$$
R\left(E_{0}\right)=A E_{0}^{1.75} \mathrm{~g} \mathrm{~cm}^{-2},
$$

where $\mathrm{A}=4.57 \times 10^{-6}$. Using Eq. (A.10), the integral is expressed by

$$
-\int_{E_{0}}^{0} \frac{d E}{(d E / d s)}=A E^{1.75} .
$$

With substituting Eq. (A.11) into Eq. (A.5), a new variable $\xi$ that equals to the energy $E$ at the top of the atmosphere $(x=0)$ can be defined in terms of $C$

$$
\xi(\mu, x, E)=\left[\frac{1}{A} C\right]^{1 / 1.75},
$$

which gives the Eq. (5). The variable $\xi$ can be used to express $\phi(C)$.

$$
\phi(C)=2 \pi \frac{d E}{d s}(\xi) J_{0}(\xi) .
$$

Then, the solution for $f$ is given in the form

$$
f(\mu, x, E)=\frac{2 \pi d E / d s(\xi)}{v d E / d s(E)} J_{0}(\xi),
$$

which satisfies the boundary condition of Eq. (A.8). The function $d E / d s$ is obtained by differentiating both sides of Eq. (A.5) with respect to $E$. In this case where the definition in Eq. (A.11) is employed, $d E / d s$ is given as

$$
\frac{d E}{d s}(y)=-\frac{y^{-0.75}}{1.75 A},
$$

where the variable $y$ denotes an arbitrary energy.

Thus, substituting Eq. (A.15) into Eq. (A.14) gives Eq. (4).

\section{References}

Banks, P. M. and A. F. Nagy, Concerning the Influence of Elastic Scattering Upon Photoelectron Transport and Escape, J. Geophys. Res., 75, 19021910, 1970.

Banks, P. M., C. R. Chappell, and A. F. Nagy, A New Model for the Interaction of Auroral Electrons With the Atmosphere: Spectral Degradation, Backscatter, Optical Emission, and lonization, J. Geophys. Res., 79, 1459-1470, 1974.

Berger, M. J., S. M. Selzer, and K. Maeda, Energy deposition by auroral electrons in the atmosphere, J. Atmos. Terr. Phys., 32, 1015-1045, 1970.

Christon, S. P., D. G. Mitchell, D. J. Williams, L. A. Frank, C. Y. Young, and T. E. Eastman, Energy Spectra of Plasma Sheet Ions and Electrons From $\sim 50 \mathrm{eV} / \mathrm{e}$ to $\sim 1 \mathrm{MeV}$ During Plasma Temperature Transitions, $J$. Geophys. Res., 93(A4), 2562-2572, 1988.

Hoshino, M., T. Mukai, T. Terasawa, and I. Shinohara, Superthermal electron acceleration in magnetic reconnelction, J. Geophys. Res., 106(A11), 25,979-25,997, 2001.

Kurihara, J., T. Abe, K. Oyama, E. Griffin, M. Kosch, A. Aruliah, K. Kauristie, Y. Ogawa, S. Komada, and N. Iwagami, Observations of the lower thermospheric neutral temperature and density in the DELTA campaign, Earth Planets Space, 58, in this issue, 1123-1130, 2006.

Luhmann, J. G., Auroral electron spectra in the atmosphere, J. Atmos. Terr. Phys., 38, 605-610, 1976.

Lummerzheim, D., M. H. Rees, and H. R. Anderson, Angular dependent transport of auroral electrons in the upper atmosphere, Planet. Space Sci., 37, 109-129, 1989.

MacDonald, W. M. and M. Walt, Distribution Function of Magnetically Confined Electrons in a Scattering Atomsphere, Ann. Phys., 15, 44-62, 1961.

Morooka, M., T. Mukai, and H. Fukunishi, Current-voltage relationship in the auroral particle acceleration region, Ann. Geophys., 22, 3641-3655, 2002.

Mozer, F. S., S. D. Bale, T. D. Phan, and J. A. Osborne, Observations of Electron Diffusion Regions at the Subsolar Magnetopause, Phys. Rev. Lett., 91, 24, 245002(1-4), 2002.

Nagy, A. F. and P. M. Banks, Photoelectron Fluxes in the Ionosphere, $J$. Geophys. Res., 75, 6260-, 1970.

Ogasawara, K., K. Asamura, T. Mukai, and Y. Saito, Avalanche Photodiodes for measurement of low-energy electrons, Nucl. Inst. and Meth. A, $\mathbf{5 4 5 , ~ 3 , ~ 7 4 4 - 7 5 2 , ~} 2005$.

Ogasawara, K, T. Takashima, K. Asamura, Y. Saito, and T. Mukai, The Effect of Depletion Layer Thickness in Avalanche Photodiodes for Measurement of Low-energy Electrons, Nucl. Inst. and Meth. A, 566, 2, 575583, 2006.

Porter, H. S., F. Varosi, and H. G. Mayr, Iterative Solution of the Multistream Transport Equation 1. Comparison with Laboratory Beam Injection Experiments, J. Geophys. Res., 92, 5933-5959, 1987.

Rees, M. H., Auroral ionization and excitation by energeticelectrons, Planet. Space Sci., 11, 1209-1218, 1963.

Solomon, S. C., Auroral particle transport using the Monte Carlo method, Geophys. Res. Lett., 20, 185-188, 1993.

Solomon, S. C., Auroral particle transport using Monte Carlo and hybrid methods, J. Geophys. Res., 106, 107-116, 2001.

Stamnes, K., Analytic Approach to Auroral Electron Transport and Energy Degradation, Planet. Scace Sci., 11, 28, 427-441, 1980.

Stamnes, K., On the Two-Stream Approach to Electron Transport and Thermalization, J. Geophys. Res., 86, 2405-2410, 1981.

Strickland, D. J., D. L. Book, T. P. Coffey, and J. A. Fedder, Transport Equation Techniques for the Deposition of Auroral Electrons, J. Geophys. Res., 88, 2755-2764, 1976.

K. Ogasawara (e-mail: keiichi@stp.isas.jaxa.jp), K. Asamura, T. Takashima, Y. Saito, and T. Mukai 